\title{
Diagnostic value of VCG combined with CDE for acute inferior MI culprit vessel
}

\author{
Zhihui Zhao, Li Jiang \\ Department of Medical Ultrasonics, Baogang Hospital, Baotou, Inner Mongolia, China
}

Received: January 16, 2019

Accepted: February 25, 2019 Online Published: March 10, 2019

DOI: $10.5430 /$ dcc.v6n1p7

URL: https://doi.org/10.5430/dcc.v6n1p7

\begin{abstract}
Objective: To explore the diagnostic value of vectorcardiography (VCG) combined with color Doppler echocardiography (CDE) for acute inferior myocardial infarction (MI) culprit vessel.

Methods: A total of 82 patients who had been clinically diagnosed as acute inferior MI and had received coronary arteriography (CAG) were selected as research objects. They received bedside VCG and CDE examinations after admission. Their culprit vessels were analyzed and compared with CAG results to evaluate the diagnostic efficiency.

Results: The detection rate of acute inferior MI culprit vessel by VCG combined with CDE was $76.83 \%$, including 18 left circumflex (LCX) branches and 45 right coronary arteries (RCA). The lower left constituent ratio of LCX was $61.11 \%$, which was significantly higher than $20.00 \%$ in RCA $(p<.05)$; the lower right constituent ratio of RCA was $71.11 \%$, which was much higher than $11.11 \%$ in LCX $(p<.05)$; the sensitivity and the accuracy of RCA culprit vessel judged with frontal plane ST-vector pointing to the lower right quadrant were $71.11 \%$ and $53.33 \%$ respectively; the diagnostic efficiency of LCX culprit vessel judged with frontal plane ST-vector pointing to the lower left quadrant was $61.11 \%$ and $50.00 \%$ respectively. The maximum amplitude of the frontal plane in LCX culprit vessel was significantly lower than that in RCA culprit vessel; and the frontal plane vector angle in LCX was significantly greater than that in RCA culprit vessel $(p<0.05)$.

Conclusions: VCG combined with CDE can effectively diagnose acute inferior MI. It has a good diagnostic efficiency by judging culprit vessels according to the frontal plane ST-vector direction.
\end{abstract}

Key Words: VCG, Echocardiography, Inferior MI, Culprit vessel, Diagnosis

\section{INTRODUCTION}

Myocardial infarction (MI), which is a type of necrosis disease in myocardial tissues, resulting from continuous myocardial ischemia, is a high risk factor for cardiac sudden death. To increase the accuracy of MI diagnosis can effectively improve the prognosis and prevent the recurrence of MI. ${ }^{[1]}$ The most commonly used MI examinations in clinical application include electrocardiography (ECG), color Doppler echocardiography (CDE), coronary arteriography (CAG) and so on. ECG is mainly used to observe the de- pression or elevation of the corresponding ST segment and T wave inversion after $\mathrm{J}$ point to judge the disease condition and diagnose the culprit vessel. However, it is difficult to diagnose untypical MI by this method. CDE, commonly adopted as a supplementary measure to ECG, can reflect the infarction size. ${ }^{[2,3]}$ Vectorcardiography (VCG) is a type of vector-based graphics, and it can reflect cardiac electric activities in the order of occurrence through projections on the frontal plane, the horizontal plane and the lateral plane. Compared with ECG, it is more informative and accurate and

\footnotetext{
*Correspondence: Zhihui Zhao; Email: zzhbghos@163.com; Address: Department of Medical Ultrasonics, Baogang Hospital, Baotou, Inner Mongolia, China.
}

Published by Sciedu Press 
can be used to diagnose culprit vessels, providing a reference for physicians for the use of diagnosis. ${ }^{[4]}$ Taking patients with acute inferior MI as research objects, this research is aimed at the diagnostic value of VCG combined with CDE for acute inferior MI culprit vessel.

\section{DATA AND METHODS}

\subsection{Case information}

A total of 82 cases of patients who were diagnosed as acute inferior MI during inpatient in our hospital from April of 2012 to October of 2017 were selected as research objects. Inclusion criteria for research objects were as follows: (1) patients who were diagnosed as acute inferior MI by means of CAG and laboratory examinations; (2) the culprit vessels were shown to be right coronary arteries (RCA) or left circumflex (LCX) branches; (3) patients who understood this research and signed the informed consent form. Exclusion criteria were as follows: (1) patients with acute inferior MI accompanying serious cardiovascular and cerebrovascular diseases and malignant tumors; (2) patients with acute inferior MI accompanying serious anterior descending artery stenosis or triple vessel coronary artery disease, which may interfere with the judgment of RCA and LCX culprit vessels; (3) patients with a past medical history of MI; (4) patients with acute inferior MI accompanying ventricular hypertrophy, intra-ventricular block, pre-excitation syndrome and other diseases which may affect ventricular depolarization vectors.

\subsection{Examination methods}

Patients were required to receive $\mathrm{CAG}$ examination in $12 \mathrm{~h}$ and VCG, CDE examinations in $48 \mathrm{~h}$ after the occurrence of disease.

\subsubsection{VCG}

With the help of synchronous 12-lead ECG workstation (Beijing Vales \& Hills Biomedical Tech. Ltd.), bedside VCG examination was performed, with Frank lead system used for scanning and recording. Simultaneously, 12-lead ECG was recorded to observe the frontal plane ST-vector angle and direction, as well as the angle and amplitude of the instantaneous vector of each $10 \mathrm{~ms}$ within $40 \mathrm{~ms}$ since QRS loop started. The diagnostic standards of inferior MI by means of VCG were: (1) frontal plane QRS loop centrifugally rotated in clockwise direction, the vector pointed to the upper right quadrant in the first $0.01 \mathrm{~s}$, and then turned to the upper left quadrant at $0.02 \mathrm{~s}$; between 0.03 and $0.04 \mathrm{~s}$, the vector slightly pointed to the upper left. Besides, the amplitude of QRS loop above $\mathrm{X}$ axis was more than $0.2 \mathrm{mV}$, and the corresponding movement time was more than $0.025 \mathrm{~s}$, with left thrust more than $0.3 \mathrm{mV}$ and the maximum angle of QRS loop less than $10^{\circ}$. (2) In the right lateral plane, the initial vector of QRS loop pointed to the upper and the anterior, with the loop rotating in clockwise direction, and stayed above $\mathrm{X}$ axis between 0.025 and $0.3 \mathrm{~s}$. (3) In the transverse plane, the loop showed no significant manifestations. (4) $\mathrm{T}$ loop was usually shown as a big circle.

\subsection{2 $C D E$}

Vivid E9 color ultrasound system (GE company), with the probe frequency of $2.5 \mathrm{MHz}$, was used to observe not only left and right ventricular ejection fractions and stroke volumes, but also E peak and A peak to judge whether ventricular wall motion remained normal and the ventricular wall turned thinner with strong intracardiac echoes. The judgment standards of culprit vessels by means of CDE: ventricular wall motion abnormality appeared in inferior wall, posterior wall, middle segment even base segment of posterior interventricular septum, which can judge that RCA was considered to be the culprit vessel; ventricular wall motion abnormality appeared in posterior wall, between base segment and middle segment of inferior wall, and motion abnormality appeared between base segment and middle segment of lateral wall, with no obvious changes in base segment of posterior interventricular septum, which can judge that LCX was considered to be the culprit vessel.

\subsection{Statistical methods}

SPSS 19.0 statistical software was applied to statistical analysis, and $\chi^{2}$ test was used in the comparison of categorical data. The measurement data were represented by $\bar{X} \pm \mathrm{s}$, with $t$-test used. The difference $p<.05$ was of statistical significance.

\section{RESUltS}

\subsection{Vector distribution of culprit vessels}

CAG results, considered as the gold standards for the determination of culprit vessels, showed that 60 RCA and 22 LCX were included in culprit vessels with infarction. There were 63 culprit vessels diagnosed as acute inferior MI by means of VCG combined with $\mathrm{CDE}$, and the detection rate was $76.83 \%$ (63/82), including 18 LCX and 45 RCA. From the aspect of ST-vector direction in different vessels, the constituent ratio of LCX with ST-vector pointing to the lower left quadrant was $61.11 \%$, which was significantly higher than that of RCA with ST-vector pointing to the lower left quadrant $(20 \%, p<.05)$; the constituent ratio of RCA with ST-vector pointing to the lower right quadrant was $71.11 \%$, which was obviously higher than that of LCX with ST-vector pointing to the lower right quadrant $(11.11 \%, p<.05)$ (see Table 1). 
Table 1. Vector distribution of culprit vessels $(n=82)$

\begin{tabular}{lllllll}
\hline \multirow{2}{*}{ Culprit Vessels } & \multicolumn{5}{c}{ ST-vector Direction } & \multirow{2}{*}{ CAG } \\
\cline { 2 - 7 } & Lower Left & Lower Right & Upper Left & Upper Right & Total \\
\hline LCX & 11 & 2 & 3 & 2 & 18 & 22 \\
RCA & $9^{*}$ & $32^{*}$ & 1 & 3 & 45 & 60 \\
\hline
\end{tabular}

Notes. In comparison with LCX, ${ }^{*} p<.05$

\subsection{Analysis on diagnostic efficiency of frontal plane ST- vector pointing to the lower right quadrant to diag- nose RCA culprit vessel}

The sensitivity of frontal-plane ST-vector pointing to the lower right quadrant to judge RCA culprit vessel was $71.11 \%$ (32/45), and the accuracy was $53.33 \%$ (32/60) (see Table 2).

Table 2. Analysis on diagnostic efficiency of frontal plane ST-vector pointing to the lower right quadrant to diagnose RCA culprit vessel $(n=60)$

\begin{tabular}{llll}
\hline \multirow{2}{*}{ Frontal Plane ST-vector } & \multicolumn{2}{c}{ CAG Results } & \multirow{2}{*}{ Total } \\
\cline { 2 - 3 } & RCA & non-RCA & \\
\hline Lower Right & 32 & 1 & 33 \\
Excluding Lower Right & 13 & 14 & 27 \\
Total & 45 & 15 & 60 \\
\hline
\end{tabular}

\subsection{Analysis on diagnostic efficiency of frontal plane ST- vector pointing to the lower left quadrant to diag- nose $L C X$ culprit vessel}

The sensitivity of frontal-plane ST-vector pointing to the lower left quadrant for the judgment of LCX culprit vessel was $61.11 \%(11 / 18)$, and the accuracy was $50 \%$ (11/22) (see Table 3).

Table 3. Analysis on diagnostic efficiency of frontal plane ST-vector pointing to the lower left quadrant to diagnose LCX culprit vessel $(n=22)$

\begin{tabular}{llll}
\hline \multirow{2}{*}{ Frontal Plane ST-vector } & \multicolumn{2}{c}{ CAG Results } & \multirow{2}{*}{ Total } \\
\cline { 2 - 3 } & LCX & non-LCX & \\
\hline Lower Left & 11 & 1 & 12 \\
Excluding Lower Left & 7 & 3 & 10 \\
Total & 18 & 4 & 22 \\
\hline
\end{tabular}

\subsection{Imaging analysis on frontal plane QRS loop by means of VCG}

The maximum frontal-plane amplitude of LCX culprit vessel was more than $0.4 \mathrm{mV}$, and the average value was $(0.86 \pm$ $0.24) \mathrm{mV}$, which was significantly lower than that of RCA culprit vessel $((1.15 \pm 0.23) \mathrm{mV}, p<.05)$; the angle of frontal-plane vector of LCX was $(25.32 \pm 5.67)^{\circ}$, which was obviously greater than that of RCA $\left((20.43 \pm 4.87)^{\circ}, p<\right.$ $.05)$.

\section{DISCUSSION}

Acute MI is one of the most common and serious coronary atherosclerotic heart diseases, in which anterior MI is prone to ventricular arrhythmias; inferior MI mostly shows decreased heart rate and atrioventricular block. ${ }^{[5]}$ Patients with the latter disease may develop cardiac rupture, arhythmia, cardiac failure, cardiogenic shock and other complications. Once acute MI occurs, it is supposed to early determine the infarction-associated artery and the stenosis zone. It is of great guiding significance to determine whether it is required to perform vascular reconstruction. ${ }^{[6]}$

Non-invasive examinations for myocardial ischemia include conventional 12-lead ECG, treadmill exercise, dynamic electrocardiogram, radionuclide myocardial tomography and so on. ECG is mainly used to observe II, III and AVF lead. Patients with infarction usually show the elevation of the corresponding ST segment or obvious depression of ST segment after J point. However, this change in ST segment is prone to transient myocardial ischemia, medication, electrolyte disturbance and other various factors. Hence, it is required to use ECG in combination with other supplementary means. ${ }^{[7]}$ Electrocardial vector refers to myocardial electrodynamic force generated during the depolarization or the repolarization on the cell membranes of the myocardial in the course of impulse. As myocardial electrodynamic force is a vector of magnitude, it is called spatial electrocardial vector, which is short for electrocardial vector. ${ }^{[8]}$ Every moment of the impulse, myocardial cells in each part of heart go through depolarization and repolarization, and then generate various electrocardial vectors with different directions. The maximum vectors of all instantaneous electrocardial vectors in a cardiac cycle are connected in the order of time, so that a closed circular motion curve is formed and called electrocardial vector loop. Conventional 12-lead ECG is considered to be the projection of VCG on each lead axis, and it is a different pattern of manifestation for the same thing. However, VCG can also reflect the change in the direction of instantaneous electrocardial vector. ${ }^{[9]}$

Xixuan Zhao et al. ${ }^{[10]}$ analyzed the relationship between inferior MI frontal plane vectors and coronary arterial culprit vessels. The change in the angle of ST vector was applied to the differentiation of infarcted coronary arteries. The results 
showed the amplitude of the maximum depolarization vector of RCA was significantly lower than that of LCX, affirming that the direction of frontal plane ST vector was of guiding significance to determine culprit vessels for patients with acute inferior MI. Yuqin Wu et al. ${ }^{[11]}$ analyzed the diagnostic efficiency of ECG, VCG and CDE. The results showed that the accuracy of each method to detect acute MI was $65.79 \%$, $26.32 \%$ and $7.89 \%$ respectively. However, for the detection of remote MI, the detection rate of each method was $18.18 \%$, $54.55 \%$ and $27.27 \%$ respectively, and it was found that a patient developed left ventricular aneurysm through VCG examination. Therefore, ECG is considered to play an irreplaceable role in the diagnosis of acute MI. Nevertheless, as to different types of MI, it is suggested to use ECG in combination with other non-invasive diagnostic methods for the diagnosis to improve the diagnostic efficiency.

VCG combined with CDE was used to judge the disease condition in the research, with CAG results taken as gold standards. The type of inferior MI was fully evaluated according to ST vector direction. The results showed that the detection rate of the combined diagnosis reached up to $76.83 \%$. In addition, the research also showed that the accuracy of frontal plane ST-vector pointing to the lower right quadrant for the judgment of RCA culprit vessel was higher than that of frontal plane ST-vector pointing to the lower left quadrant for the judgment of LCX culprit vessel; and the maximum amplitude of frontal plane of RCA culprit vessel was significantly higher than that of LCX. All these were consistent with the research made by Yuqin Wu et al. ${ }^{[11]}$ When LCX or RCA was blocked, frontal plane ST-vector might point to the lower left, the lower right, the upper left and the upper right quadrants. If frontal plane ST-vector pointed to the lower left quadrant, it conformed with LCX lesion characteristics; if frontal plane ST-vector pointed to the lower right quadrant, it conformed with RCA lesion characteristics. Therefore, the direction of frontal plane ST-vector can be used as a reference indicator for the judgment of culprit vessels.

\section{Conclusions}

In conclusion, frontal plane ST-vector direction of VCG can be used to judge culprit vessels, and CDE is applied to the observation of chamber morphology and ventricular wall motion in different segments to judge culprit vessels. CDE in combination with VCG can significantly improve the diagnostic efficiency of acute inferior MI culprit vessel. It is recommended to apply combined diagnosis to the detection of acute inferior MI culprit vessel.

\section{CONFliCts of InTEREST Disclosure}

The authors declare they have no conflicts of interest.

\section{REFERENCES}

[1] Prab hu SD, Frangogiannis NG. The biological basis for cardiac repair after myocardial infarction: from inflammation to fibrosis. Circulation Research. 2016; 119(1): 91-112. PMid:27340270. https://doi.org/10.1161/CIRCRESAHA.116.303577

[2] Panayi G, Wieringa WG, Alfredsson J, et al. Computed tomography coronary angiography in patients with acute myocardial infarction and normal invasive coronary angiography. BMC Cardiovascular Disorders. 2016; 16(1): 1-8. PMid:27142217. https: //doi.org/10.1186/s12872-016-0254-y

[3] Juying C, Hongyu W. Prognosis evaluation of acute MI patients by multiple electrocardial and cardiac ultrasound non-invasive indexes. Chinese Journal of Integrative Medicine on Cardio/Cerebrovascular Disease. 2016; 14(13): 1523-1525.

[4] Pastore CA, Samesima N, Filho HG, et al. Controversial and similar aspects of the Brugada and $\mathbf{J}$ wave patterns: the vectorcardiogram point of view-Revision 2. Journal of Electrocardiology. 2016; 49(3): 439. PMid:27133525. https://doi.org/10.1016/j.jelectro card.2016.03.018

[5] Correa R, Arini PD, Correa LS, et al. Identification of patients with myocardial infarction vectorcardiographic and electrocardiographic analysis. Methods of Information in Medicine. 2016; 55(3): 242-249. PMid:27063981. https://doi.org/10.3414/ME15-01-0101

[6] Hongqi L. Diagnostic analysis on the electrocardiogram of acute MI. World Latest Medicine Information. 2017; 17(28): 146-148.

[7] Stare V, Schlegel TT. Delineation of QRS offset by instantaneous changes in ECG vector angle can improve detection of acute inferior myocardial infarctions. Journal of Electrocardiology. 2016; 49(3): 337-344. PMid:26979381. https://doi.org/10.1016/j.jele ctrocard.2016.02.019

[8] Hailing C. Research on diagnostic value of ECG combined with VCG for myocardial ischemia of coronary heart diseases. Chinese Journal of Integrative Medicine on Cardio/Cerebrovascular Disease. 2016; 14(22): 2666-2668.

[9] Zhishan J, Yongmei F, Chunxia X. New progress in clinical application and research of vectorcardiogram. Journal of Practical Electrocardiology JS. 2016; 25(2): 132-137.

[10] Xixuan Z, Weixin Z, Yongquan W, et al. The relationship between inferior wall myocardial infarction and the culprit lesion of coronary artery. Journal of Clinical Eletrocardiology. 2016; 25(3): 183-186.

[11] Yuqin W, Wei P. Diagnostic value of ECG, VCG and CDE for myocardial infarction. Journal of Clinical Medicine in Practice. 2017; 21(21): 79-80. 